\title{
Age-specific impact on the survival of gastric cancer patients with distant metastasis: an analysis of SEER database
}

\author{
Xinxing Li ${ }^{1, *}$, Weijun Wang ${ }^{1, *}$, Canping Ruan ${ }^{1, *}$, Yi Wang ${ }^{1, *}$, Haolu Wang ${ }^{2}$, Xiaowen \\ Liang', Yanping Sun ${ }^{1}$ and Zhiqian $\mathrm{Hu}^{1}$ \\ ${ }^{1}$ Department of General Surgery, Changzheng Hospital, The Second Military Medical University, Shanghai, 200003, China \\ ${ }^{2}$ Therapeutics Research Centre, School of Medicine, The University of Queensland, Princess Alexandra Hospital, Woolloongabba, \\ QLD 4102, Australia \\ *These authors have contributed equally to this work \\ Correspondence to: Xiaowen Liang, email: x.liang@uq.edu.au \\ Yanping Sun, email: sunyanping2003@163.com \\ Zhiqian Hu, email: huzhiq163@163.com
}

Keywords: gastric cancer, distant metastasis, age, survival

Received: December 08, $2016 \quad$ Accepted: August 28, $2017 \quad$ Published: September 28, 2017

Copyright: Li et al. This is an open-access article distributed under the terms of the Creative Commons Attribution License 3.0 (CC BY 3.0), which permits unrestricted use, distribution, and reproduction in any medium, provided the original author and source are credited.

\section{ABSTRACT}

The age-specific impact on the survival of gastric cancer patients with distant metastasis is still unclear. In this study, we identified 11, 299 gastric cancer patients with distant metastasis between 2004 and 2013 from Surveillance, Epidemiology, and End Results population-based dataset. Patients were divided into young $(\leq 60)$ and elderly groups $(>60)$. Kaplan-Meier methods and multivariable Cox regression were used for the analysis of long-term survival outcomes and risk factors. There were significant differences between the two groups in terms of race, primary site, grade, histologic type, surgery, marital status and clinical $T$ stage $(P<0.05)$. The 1- and 3-year cancer specific survival rates were $29.0 \%$ and $6.2 \%$ in young group and $22.8 \%$ and $4.8 \%$ in elderly group in both univariate $\left(X^{2}=116.430, P<0.001\right)$ and multivariate analysis $(P<0.001)$. Young patients had significantly better $1-$ and 3-year cancer specific survival than elderly patients in each $\mathrm{T}$ stage. Age was further validated as an independent survival factor in all T stages $\left(T 1, T 2, T 3, T 4\right.$ and $T_{x}$ $P<0.05)$. In conclusion, age was an independent prognostic factor for gastric cancer patients with distant metastasis.

\section{INTRODUCTION}

Gastric cancer (GC) is the fifth most common malignancy worldwide and the third leading cause of cancer deaths [1-3]. Despite the improvements in diagnosis and treatment, the 5-year overall survival (OS) rate for advanced GC is still below 30\% [4-5]. Age has been recognized as an important predictor of prognosis in many cancers [6-7]. The prevalence of GC increases with age, and the peak incidence is in old population of 60-70 years [8-9]. Clarification of the relationship between age and GC survival could reveal the impact of age on cancer prognosis and improve treatment efficacy [10].
Some studies reported that young GC patients usually had advanced stage and undifferentiated tumors [11-12]. Song et al. [8] argued that the prognosis of GC varied with age, and young patients had a higher survival rate after surgery compared to elderly patients. Similar results were also found in Li's study [6], where young patients with colorectal cancer after surgery had a higher cancer specific survival (CSS) rate than elderly ones [6]. Although the survival and age at diagnosis in $\mathrm{GC}$ has been investigated $[8-9,13]$, age-specific impact on the survival in GC patients with distant metastasis (M1) is still unclear. In this study, we compared the pathological characteristics and prognostic outcomes of GC with M1 in young patients 
with elderly ones based on Surveillance, Epidemiology, and End Results (SEER) population-based data.

\section{RESULTS}

\section{Patient characteristics}

We identified 11, 299 GC patients with M1 diagnosed between 2004 and 2013 of the known age $(\geq 18)$. In this study, we classified the patients into two groups: young $(\leq 60)$ and elderly $(>60)$ patients, including 7,128 $(63.09 \%)$ males and 4,171 (36.91\%) females. The average follow-up period was 5 months. Patient demographics and pathological features were summarized in Table 1.

\section{Characteristics of GC patients}

There were significant differences between the two groups in terms of race, primary site, grade, histologic type, surgery, marital status and clinical $\mathrm{T}$ stage $(\mathrm{P}<0.05)$. Compared to elderly ones, young GC patients with M1 had more undifferentiated grade $(66.0 \%$ VS $57.7 \%, \mathrm{P}<0.05)$, more signet-ring cancer $(35.0 \%$ VS $20 \%, \mathrm{P}<0.05)$, and more stage T3 and T4 (9.8\% VS 8.2\%, 22.4\% VS $19.0 \%$; $\mathrm{P}<0.05)$. Except for married patients in young and elderly groups $(58.8 \%$ and $58.9 \%)$, most of young ones were single/separated/unmarried (26.7\%), while most of elderly were widowed (19.3\%). As shown in Table 1, no significant differences were found between two groups in years of diagnosis $(\mathrm{P}=0.955)$ and $\operatorname{sex}(\mathrm{P}=0.086)$.

\section{Impact of age on GC survival outcomes}

As shown in Tables 2 and 3 and Figure 2, the 1- and 3 -year CCS rates of GC were $29.0 \%$ and $6.2 \%$ in young group, and $22.8 \%$ and $4.8 \%$ in elderly group, which had significant difference using univariate $\left(X^{2}=116.430\right.$, $\mathrm{P}<0.001$ ) and multivariate analysis (young group as ref., HR $=0.808,95 \% \mathrm{CI}: 0.773 \sim 0.844, \mathrm{P}<0.001$ ). Year of diagnosis, sex, race, primary site, grade, histological type, surgery, marital status and clinical $\mathrm{T}$ stage were identified as significant risk factors for poor survival by univariate analysis (Table 2 and Figure $1, \mathrm{P}<0.05$ ). Female, black and American Indian/Alaska Native and widowed GC patients also had shorter survival periods (Table 2 and Figure 1). As shown in Table 2, the 1- and 3 -year CCS rates of patients in stage T1 (25.3\% and 5.1\%) were lower than that of in T2 $(35.2 \%$ and $8.4 \%)$ and T3 (34.7\% and $8.8 \%)$, but higher than that in T4 $(22.8 \%$ and $4.9 \%)$ and $\mathrm{Tx}(21.0 \%$ and $3.8 \%)$. Multivariate analysis with Cox regression revealed that year of diagnosis, age, race, primary site, grade, histological type, surgery, marital status and pathological $\mathrm{T}$ stage were independent prognostic factors (Table $3, \mathrm{P}<0.05$ ).

\section{Stratified analysis of age on GC survival based on T stage}

We then further analyzed the effect of age on 1- and 3-year CSS at each clinical T stage. We found that young patients had significantly better 1 - and 3-year CSS than elderly patients in each $\mathrm{T}$ stage (Table $4, \mathrm{P}<0.05$ ). Age was further validated as an independent survival factor in multivariate Cox regression at T1 stage (elderly, HR: 0.824, 95\%CI: 0.742 0.915; $\mathrm{P}<0.001$ ), $\mathrm{T} 2$ stage (elderly, HR: $0.756,95 \%$ CI: $0.680 \sim 0.841 ; \mathrm{P}<0.001)$, T3 stage (elderly, HR: $0.818,95 \%$ CI: 0.713 0.938; $\mathrm{P}=0.004$ ), T4 stage (elderly, HR: 0.811, 95\%CI: 0.742 0.886; $\mathrm{P}<0.001$ ) and Tx stage (elderly, HR: 0.784, 95\%CI: 0.733 0.838; $\mathrm{P}<0.001$ ) patients (Table 4).

\section{DISCUSSION}

Despite advancement of diagnosis and treatment of $\mathrm{GC}$, the prognosis remains poor with a 5-year OS of less than $30 \%$ in most countries $[4-5,14]$. In China, GC is the second leading cause of cancer death, and the current 5 year CSS is low because more than $80 \%$ of patients are diagnosed at an advanced stage [15-16]. Age is considered as one of the independent factors of several cancers [6$9,12]$. Investigation of important prognostic factors of GC development could further understand and improve the treatment of the advanced disease. We identified 11, 299 GC patients with M1 diagnosed between 2004 and 2013 with a known age based on SEER population-based data. The current definition of elderly patients remains controversial. Some studies used the cutoff age of 50 years, while others used 30 years or 45 years $[6,8-9,17]$. In this study, we divided GC patients into young $(\leq 60)$ and elderly $(>60)$ groups according to recent publications and the new age subsection-standard of the United Nations world health organization [8-9].

Recently, some studies have investigated the prognostic outcome of GC in young patients in comparison to the elderly, but yielded inconclusive results [18-19]. It has been suggested that young patients suffered worse survival due to the characteristics of themselves and different tumor behavior [20]. Chen et al. [9] reported that between 56 and 65 years have more favorable clinicopathologic characteristics and better CSS than the other groups in operable gastric cancer patients. While Song et al. [8] argued that the prognosis of GC varied with age, and young patients suffered a higher survival rate after surgery compared to elderly patients. In our study, we found that young GC patients with M1 had a higher CCS rate compared to elderly ones. The 1 - and 3-year CCS rates of GC were $29.0 \%$ and $6.2 \%$ in young group and $22.8 \%$ and $4.8 \%$ in elderly group, which had significant difference by univariate $\left(X^{2}=116.430\right.$, 
Table 1: Characteristics of GC patients with M1 from SEER database

\begin{tabular}{|c|c|c|c|c|c|}
\hline \multirow[t]{2}{*}{ Characteristic } & Total (n) & Young $(\leq 60)$ & Elderly $(>60)$ & $\mathrm{X}^{2}$ & P value \\
\hline & 11299 & 5004 & 6295 & & \\
\hline Media follow up (month) & 5 & 6 & 4 & & \\
\hline Years of diagnosis & & & & 0.003 & 0.955 \\
\hline 2004-2008 & 5549 & $2456(49.1)$ & $3093(49.1)$ & & \\
\hline $2009-2013$ & 5750 & $2548(50.9)$ & $3202(50.9)$ & & \\
\hline Sex & & & & 2.953 & 0.086 \\
\hline Male & 7128 & $3113(62.2)$ & $4015(63.8)$ & & \\
\hline Female & 4171 & $1891(37.8)$ & $2280(36.2)$ & & \\
\hline Race & & & & 12.056 & 0.007 \\
\hline White & 8305 & $3617(72.3)$ & $4688(74.5)$ & & \\
\hline Black & 1434 & $664(13.3)$ & $770(12.2)$ & & \\
\hline $\begin{array}{l}\text { American Indian/Alaska } \\
\text { Native }\end{array}$ & 117 & $66(1.3)$ & $51(0.8)$ & & \\
\hline $\begin{array}{l}\text { Asian or Pacific } \\
\text { Islander }\end{array}$ & 1443 & $657(13.1)$ & $786(12.5)$ & & \\
\hline Primary site & & & & 25.720 & 0.001 \\
\hline Cardia, NOS & 3552 & $1520(30.4)$ & $2032(32.3)$ & & \\
\hline Fundus of stomach & 463 & 197(3.9) & $266(4.2)$ & & \\
\hline Body of stomach & 1021 & $470(9.4)$ & $551(8.8)$ & & \\
\hline Gastric antrum & 1708 & $739(14.8)$ & $969(15.4)$ & & \\
\hline Pylorus & 233 & $85(1.7)$ & $148(2.4)$ & & \\
\hline $\begin{array}{l}\text { Lesser curvature of } \\
\text { stomach NOS }\end{array}$ & 642 & $269(5.4)$ & $373(5.9)$ & & \\
\hline $\begin{array}{l}\text { Greater curvature of } \\
\text { stomach NOS }\end{array}$ & 380 & $190(3.8)$ & $190(3.0)$ & & \\
\hline $\begin{array}{l}\text { Overlapping lesion of } \\
\text { stomach }\end{array}$ & 1061 & $508(10.2)$ & $553(8.8)$ & & \\
\hline Stomach, NOS & 2239 & $1026(20.5)$ & $1213(19.3)$ & & \\
\hline Grade & & & & 107.261 & 0.000 \\
\hline $\begin{array}{l}\text { Well/Moderately } \\
\text { differentiated }\end{array}$ & 1929 & $668(13.3)$ & $1261(20.0)$ & & \\
\hline $\begin{array}{l}\text { Poorly differentiated/ } \\
\text { Undifferentiated }\end{array}$ & 6933 & $3301(66.0)$ & $3632(57.7)$ & & \\
\hline Unknown & 2437 & $1035(20.7)$ & $1042(22.3)$ & & \\
\hline Histologic type & & & & 341.140 & 0.000 \\
\hline Adenocarcinoma, NOS & 7338 & $2813(56.2)$ & $4525(71.9)$ & & \\
\hline Carcinoma & 951 & $441(8.8)$ & $510(8.1)$ & & \\
\hline $\begin{array}{l}\text { Signet ring cell } \\
\text { carcinoma }\end{array}$ & 3010 & $1750(35.0)$ & $1260(20.0)$ & & \\
\hline
\end{tabular}

(Continued) 


\begin{tabular}{|c|c|c|c|c|c|}
\hline \multirow[t]{2}{*}{ Characteristic } & Total (n) & Young $(\leq 60)$ & Elderly (>60) & $\mathbf{X}^{2}$ & P value \\
\hline & 11299 & 5004 & 6295 & & \\
\hline Surgery & & & & 13.492 & 0.000 \\
\hline Yes & 1693 & $819(16.4)$ & $874(13.9)$ & & \\
\hline No & 9606 & $4185(83.6)$ & $5421(86.1)$ & & \\
\hline Marital status & & & & 1235.527 & 0.000 \\
\hline Married & 6649 & $2944(58.8)$ & $3705(58.9)$ & & \\
\hline Divorced & 978 & $455(9.1)$ & $523(8.3)$ & & \\
\hline Widowed & 1308 & $90(1.8)$ & $1218(19.3)$ & & \\
\hline $\begin{array}{l}\text { Single/separated/ } \\
\text { unmarried }\end{array}$ & 1927 & $1336(26.7)$ & $591(9.4)$ & & \\
\hline Unknown & 437 & $179(3.6)$ & $258(4.1)$ & & \\
\hline T stage & & & & 44.531 & 0.000 \\
\hline $\mathrm{T} 1$ & 1814 & $730(14.6)$ & $1084(17.2)$ & & \\
\hline $\mathrm{T} 2$ & 1784 & $809(16.2)$ & $975(15.5)$ & & \\
\hline $\mathrm{T} 3$ & 1010 & $492(9.8)$ & $518(8.2)$ & & \\
\hline $\mathrm{T} 4$ & 2316 & $1123(22.4)$ & 1193(19.0) & & \\
\hline $\mathrm{T}_{\mathrm{x}}$ & 4375 & $1850(37.0)$ & $2525(40.1)$ & & \\
\hline
\end{tabular}

Table 2: Univariate survival analyses of GC patients with M1

\begin{tabular}{|c|c|c|c|c|c|}
\hline Variable & Total (n) & 1-year CSS & 3-year CSS & $\log \operatorname{rank} x^{2}$ & P value \\
\hline Years of diagnosis & & & & 18.913 & 0.000 \\
\hline 2004-2008 & 5549 & $24 \%$ & $4.8 \%$ & & \\
\hline 2009-2013 & 5750 & $27.2 \%$ & $6.3 \%$ & & \\
\hline Sex & & & & 5.845 & 0.016 \\
\hline Male & 7128 & $26.3 \%$ & $5.7 \%$ & & \\
\hline Female & 4171 & $24.3 \%$ & $4.9 \%$ & & \\
\hline Age & & & & 116.430 & 0.000 \\
\hline Young & 5004 & $29.0 \%$ & $6.2 \%$ & & \\
\hline Elderly & 6295 & $22.8 \%$ & $4.8 \%$ & & \\
\hline Race & & & & 19.193 & 0.000 \\
\hline White & 8305 & $25.3 \%$ & $5.2 \%$ & & \\
\hline Black & 1434 & $23.1 \%$ & $4.8 \%$ & & \\
\hline $\begin{array}{l}\text { American Indian/ } \\
\text { Alaska Native }\end{array}$ & 117 & $23.2 \%$ & $4.8 \%$ & & \\
\hline $\begin{array}{l}\text { Asian or Pacific } \\
\text { Islander }\end{array}$ & 1443 & $29.8 \%$ & $7.2 \%$ & & \\
\hline Primary site & & & & 162.320 & 0.000 \\
\hline Cardia, NOS & 3552 & $29.2 \%$ & $6.5 \%$ & & \\
\hline
\end{tabular}

(Continued) 


\begin{tabular}{|c|c|c|c|c|c|}
\hline Variable & Total (n) & 1-year CSS & 3-year CSS & $\log \operatorname{rank} x^{2}$ & P value \\
\hline $\begin{array}{l}\text { Fundus of } \\
\text { stomach }\end{array}$ & 463 & $24.6 \%$ & $4.8 \%$ & & \\
\hline Body of stomach & 1021 & $25.5 \%$ & $4.8 \%$ & & \\
\hline Gastric antrum & 1708 & $27.4 \%$ & $6.9 \%$ & & \\
\hline Pylorus & 233 & $25.7 \%$ & $6.4 \%$ & & \\
\hline $\begin{array}{l}\text { Lesser curvature } \\
\text { of stomach NOS }\end{array}$ & 642 & $30.3 \%$ & $7.0 \%$ & & \\
\hline $\begin{array}{l}\text { Greater curvature } \\
\text { of stomach NOS }\end{array}$ & 380 & $26.0 \%$ & $4.8 \%$ & & \\
\hline $\begin{array}{c}\text { Overlapping } \\
\text { lesion of stomach }\end{array}$ & 1061 & $21.2 \%$ & $4.1 \%$ & & \\
\hline Stomach, NOS & 2239 & $18.9 \%$ & $3.1 \%$ & & \\
\hline Grade & & & & 66.031 & 0.000 \\
\hline $\begin{array}{l}\text { Well/Moderately } \\
\text { differentiated }\end{array}$ & 1929 & $32.3 \%$ & $7.9 \%$ & & \\
\hline $\begin{array}{l}\text { Poorly } \\
\text { differentiated/ } \\
\text { Undifferentiated }\end{array}$ & 6933 & $24.9 \%$ & $4.9 \%$ & & \\
\hline Unknown & 2437 & $22.0 \%$ & $4.9 \%$ & & \\
\hline Histologic type & & & & 11.442 & 0.003 \\
\hline $\begin{array}{l}\text { Adenocarcinoma, } \\
\text { NOS }\end{array}$ & 7338 & $26.5 \%$ & $6.0 \%$ & & \\
\hline Carcinoma & 951 & $23.4 \%$ & $5.1 \%$ & & \\
\hline $\begin{array}{l}\text { Signet ring cell } \\
\text { carcinoma }\end{array}$ & 3010 & $23.8 \%$ & $4.1 \%$ & & \\
\hline Surgery & & & & 313.299 & 0.000 \\
\hline Yes & 1693 & $41.6 \%$ & $11.7 \%$ & & \\
\hline No & 9606 & $22.6 \%$ & $4.2 \%$ & & \\
\hline Marital status & & & & 119.561 & 0.000 \\
\hline Married & 6649 & $27.1 \%$ & $5.9 \%$ & & \\
\hline Divorced & 978 & $25.3 \%$ & $3.3 \%$ & & \\
\hline Widowed & 1308 & $17.7 \%$ & $3.3 \%$ & & \\
\hline $\begin{array}{l}\text { Single/separated/ } \\
\text { unmarried) }\end{array}$ & 1927 & $24.4 \%$ & $5.8 \%$ & & \\
\hline Unknown & 437 & $30.3 \%$ & $7.0 \%$ & & \\
\hline T stage & & & & 252.356 & 0.000 \\
\hline $\mathrm{T} 1$ & 1814 & $25.3 \%$ & $5.1 \%$ & & \\
\hline $\mathrm{T} 2$ & 1784 & $35.2 \%$ & $8.4 \%$ & & \\
\hline $\mathrm{T} 3$ & 1010 & $34.7 \%$ & $8.8 \%$ & & \\
\hline $\mathrm{T} 4$ & 2316 & $22.8 \%$ & $4.9 \%$ & & \\
\hline $\mathrm{T}_{\mathrm{X}}$ & 4375 & $21.0 \%$ & $3.8 \%$ & & \\
\hline
\end{tabular}


Table 3: Multivariate Cox model analyses of prognostic factors of GC patients with M1

\begin{tabular}{|c|c|c|c|}
\hline Variable & HR & $95 \% \mathrm{CI}$ & P value \\
\hline Years of diagnosis & & & 0.000 \\
\hline 2004-2008 & & Ref & \\
\hline $2009-2013$ & 1.115 & $(1.070 \sim 1.161)$ & 0.000 \\
\hline Sex & & & 0.900 \\
\hline Male & & Ref & \\
\hline Female & 1.035 & $(0.989 \sim 1.082)$ & 0.136 \\
\hline Age & & & 0.000 \\
\hline Young & & Ref & \\
\hline Elderly & 0.808 & $(0.773 \sim 0.844)$ & 0.000 \\
\hline Race & & & 0.008 \\
\hline White & & Ref & \\
\hline Black & 1.104 & $(1.037 \sim 1.175)$ & 0.002 \\
\hline American Indian/Alaska Native & 1.139 & $(1.050 \sim 1.235)$ & 0.002 \\
\hline Asian or Pacific Islander & 1.281 & $(1.045 \sim 1.570)$ & 0.017 \\
\hline Primary site & & & 0.000 \\
\hline Cardia, NOS & & Ref & \\
\hline Fundus of stomach & 0.754 & $(0.709 \sim 0.801)$ & 0.000 \\
\hline Body of stomach & 0.838 & $(0.751 \sim 0.935)$ & 0.002 \\
\hline Gastric antrum & 0.842 & $(0.776 \sim 0.913)$ & 0.000 \\
\hline Pylorus & 0.855 & $(0.798 \sim 0.917)$ & 0.000 \\
\hline Lesser curvature of stomach NOS & 0.918 & $(0.792 \sim 1.062)$ & 0.250 \\
\hline Greater curvature of stomach NOS & 0.750 & $(0.680 \sim 0.828)$ & 0.000 \\
\hline Overlapping lesion of stomach & 0.854 & $(0.758 \sim 0.962)$ & 0.009 \\
\hline Stomach, NOS & 0.950 & $(0.877 \sim 1.029)$ & 0.207 \\
\hline Grade & & & 0.001 \\
\hline Well/Moderately differentiated & & Ref & \\
\hline Poorly differentiated/Undifferentiated & 0.880 & $(0.822 \sim 0.943)$ & 0.000 \\
\hline Unknown & 1.044 & $(0.991 \sim 1.099)$ & 0.103 \\
\hline Histologic type & & & 0.003 \\
\hline Adenocarcinoma, NOS & & Ref & \\
\hline Carcinoma & 0.945 & $(0.899 \sim 0.995)$ & 0.30 \\
\hline Signet ring cell carcinoma & 1.003 & $(0.926 \sim 1.086)$ & 0.946 \\
\hline Surgery & & & 0.000 \\
\hline Yes & & Ref & \\
\hline No & 0.638 & $(0.597 \sim 0.681)$ & 0.000 \\
\hline Marital status & & & 0.000 \\
\hline
\end{tabular}

(Continued) 


\begin{tabular}{lccc}
\hline Variable & HR & $\mathbf{9 5 \% C I}$ & P value \\
\hline Married & & Ref & \\
Divorced & 1.076 & $(0.963 \sim 1.201)$ & 0.196 \\
Widowed & 1.234 & $(1.087 \sim 1.401)$ & 0.001 \\
Single/separated/unmarried) & 1.371 & $(1.211 \sim 1.552)$ & 0.000 \\
Unknown & 1.194 & $(1.061 \sim 1.344)$ & 0.003 \\
T stage & & & 0.000 \\
T1 & & Ref & 0.008 \\
T2 & 0.922 & $(0.868 \sim 0.979)$ & 0.000 \\
T3 & 0.790 & $(0.740 \sim 0.842)$ & 0.000 \\
T4 & 0.852 & $(0.785 \sim 0.942)$ & 0.962 \\
$\mathrm{~T}_{\mathrm{X}}$ & 0.999 & $(0.944 \sim 1.056)$ & \\
\hline
\end{tabular}

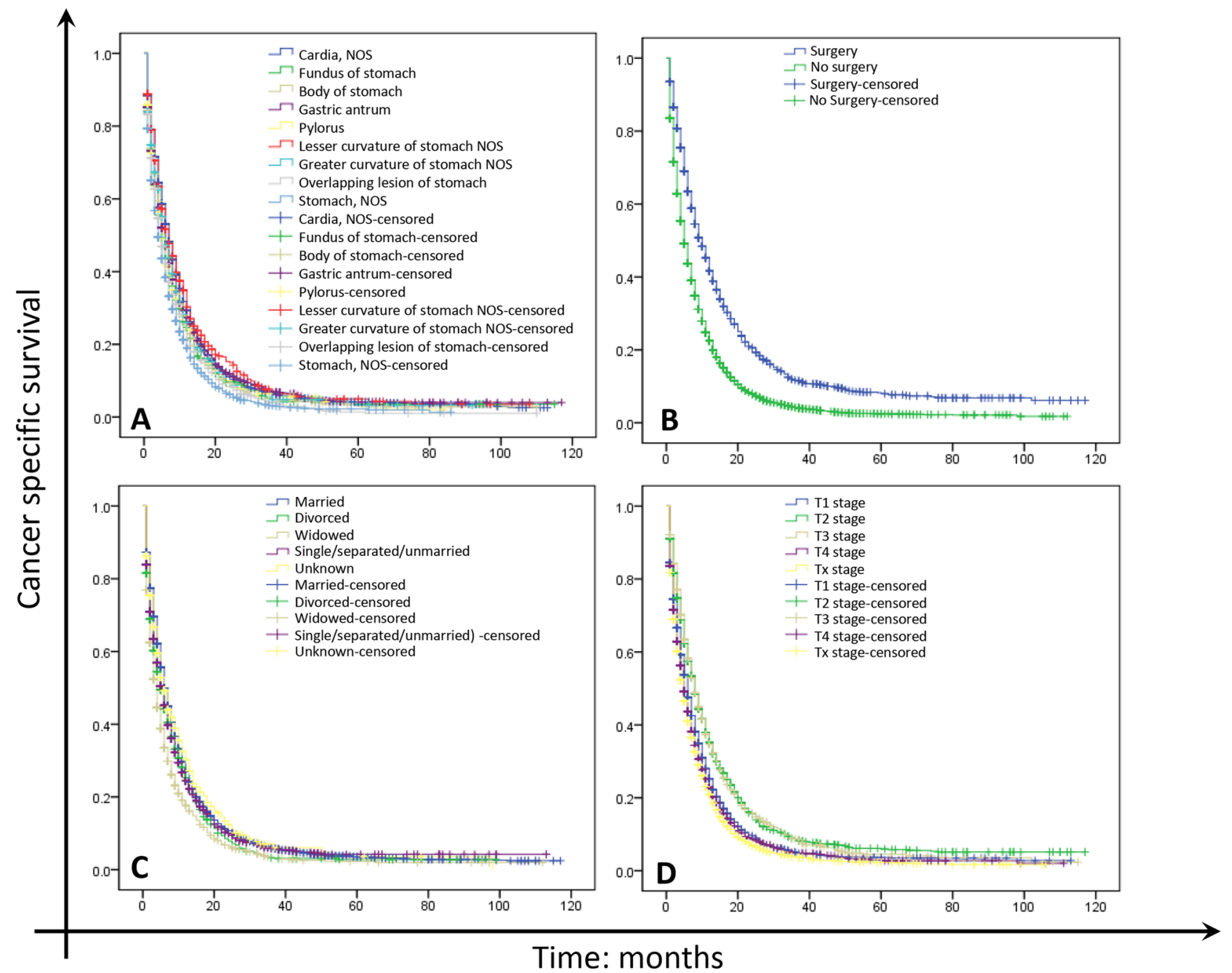

Figure 1: Survival curves in GC patients with M1 of different primary site, surgical treatment, marital status and T stage. (A) Primary site. $\mathrm{X}^{2}=162.320, \mathrm{P}<0.001$; (B) Surgery. $\mathrm{X}^{2}=313.299, \mathrm{P}<0.001$; (C) Marital status. $\mathrm{X}^{2}=119.561, \mathrm{P}<0.001$; (D) $\mathrm{T}$ stage. $\mathrm{X}^{2}=252.356, \mathrm{P}<0.001$. 
$\mathrm{P}<0.001$ ) and multivariate analysis (young group as ref., $\mathrm{HR}=0.808$, 95\%CI: 0.773 0.844) $(\mathrm{P}<0.001)$. It might be attributed to two main reasons. One could be explained by that the poor tolerance of extensive lymphadenectomy and standardized chemotherapy [21-22]. Clinicians are more likely to provide all remedial options for young patients since they have the better health condition and tolerance of chemotherapy [31]. The other reason was that young patients usually have better tolerance of surgery and better recovery [23-24]. Although some ones thought that oldness would another reason for affecting long-time survival, most of GC patients with distant metastasis died in 1 year and 3-year CSS was less than 10\%.

Another interesting finding was that compared to elderly ones, young GC patients with M1 had characteristics of more poor or undifferentiated grade $(66.0 \%$ VS $57.7 \%, \mathrm{P}<0.05)$, more signet-ring cancer $(35.0 \%$ VS $20 \%, \mathrm{P}<0.05)$, more stage T3 and T4 $(9.8 \%$ VS $8.2 \%, 22.4 \%$ VS $19.0 \%$; $\mathrm{P}<0.05)$. Li et al. [6] also found young patients presented higher proportions of unfavorable behavior as well as advanced stage disease. In contrast, it was noted that that young patients suffered worse survival due to the personal characteristics and different tumor behavior [20]. It is well known that mucinous, signet-ring and poorly differentiated tumors tend to have a poorer prognosis compared to well and moderately differentiated tumors [25]. It is thought that gastric cancer results from a combination of environmental factors and an accumulation of specific genetic alterations. The genetic information of young patients is different from that which leads to sporadic carcinomas at an older age. And there is a tendency of late diagnosis of the disease in young patients [26-27].

In addition, we found that except for married ones in young and elderly groups (58.8\% and 58.9\%), most of young GC patients were single/separated/unmarried $(26.7 \%)$, while most of elderly were widowed (19.3\%) who had the shortest survivals. Li et al. [28] selected 112, 776 colorectal cancer from SEER data and found unmarried patients were at greater risk of cancer specific mortality while widowed patients were at the highest risk of death than the other groups. Jin et al. [29] suggested that marriage had a protective effect against undertreatment and cause-specific mortality in GC. It might be attributed to that widowed patients lack of social and connubial support [30] and psychosocial distress [31]. Widowed cancer patients showed more distress, depression, and anxiety than married counterparts, which might be attributed to that spouse could share the emotional burden and provide appropriate his/her support [32]. Depression or/and nonadherence have been found to be directly correlated to widowed cancer individuals [33]. It was reported that depression was related to VEGF, stimulating endothelial cell migration,
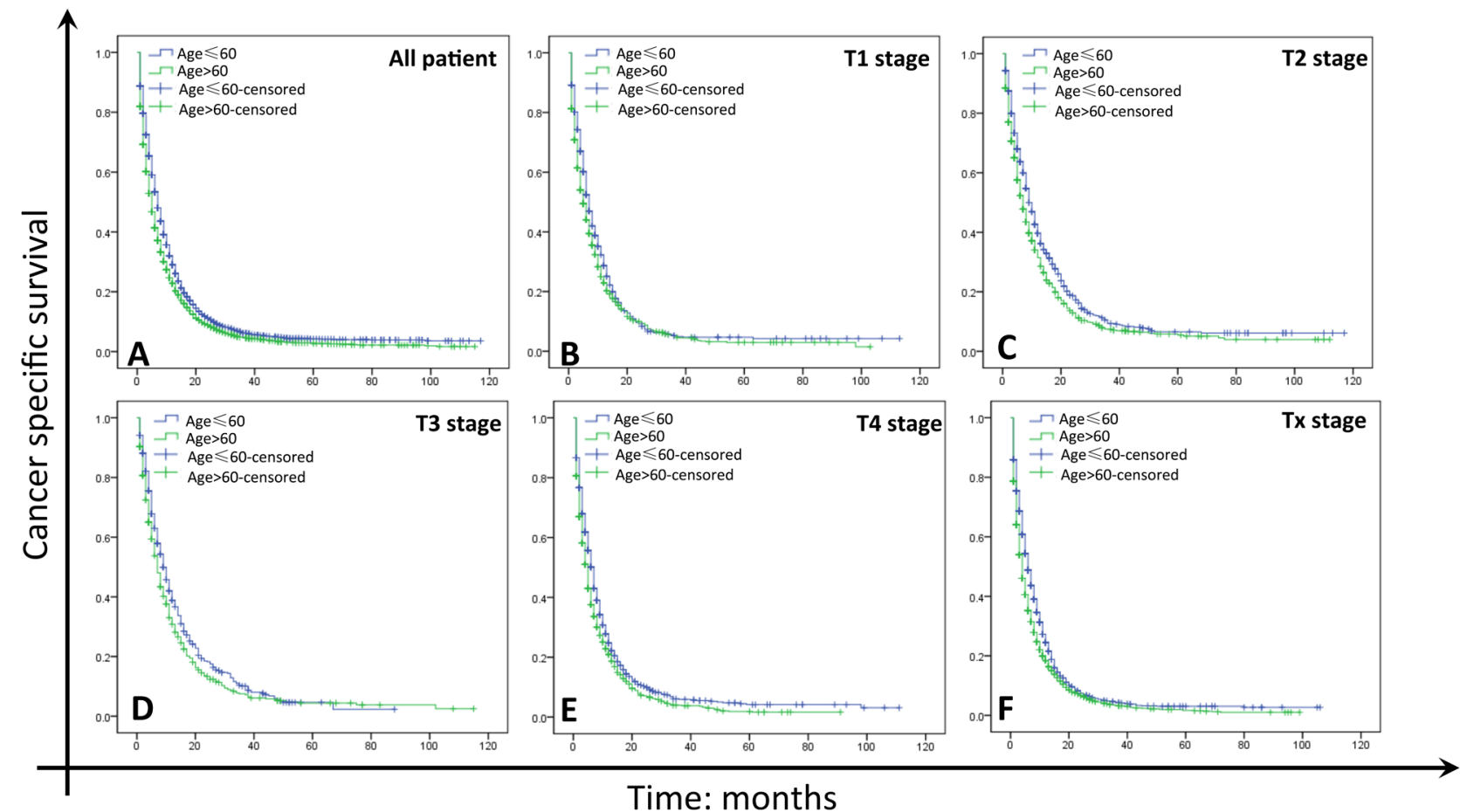

Time: months

Figure 2: Survival curves in GC patients with M1 of different age. (A) All patients. Young group vs. Elderly group, $\mathrm{X}^{2}=$ $116.430, \mathrm{P}<0.001$; (B) T1 stage. Young group vs. Elderly group, $\mathrm{X}^{2}=9.817, \mathrm{P}=0.002$; (C) $\mathrm{T} 2$ stage. Young group vs. Elderly group, $\mathrm{X}^{2}=$ 20.034, $\mathrm{P}<0.001$; (D) T3 stage. Young group vs. Elderly group, $\mathrm{X}^{2}=8.278, \mathrm{P}=0.004$; (E) T4 stage. Young group vs. Elderly group, $\mathrm{X}^{2}=$ $22.753, \mathrm{P}<0.001 ;$ (F) Tx stage. Young group vs. Elderly group, $\mathrm{X}^{2}=51.779, \mathrm{P}<0.001$. 
Table 4: Univariate and multivariate analysis of age on CSS of different $\mathrm{T}$ stages

\begin{tabular}{|c|c|c|c|c|c|c|c|}
\hline \multirow[t]{2}{*}{ Variable } & \multirow[t]{2}{*}{ Total (n) } & \multirow[t]{2}{*}{ 1-year CSS } & \multirow[t]{2}{*}{ 3-year CSS } & \multicolumn{2}{|c|}{ Univariate analysis } & \multicolumn{2}{|c|}{ Multivariate analysis } \\
\hline & & & & $\begin{array}{c}\log \text { rank } \\
x^{2}\end{array}$ & $P$ value & HR $(95 \% \mathrm{CI})$ & $\begin{array}{c}\mathrm{P} \\
\text { value }\end{array}$ \\
\hline T1 & 1814 & & & 9.817 & 0.002 & & \\
\hline \multicolumn{8}{|l|}{ Age } \\
\hline Young & 730 & $28.7 \%$ & $5.2 \%$ & & & Ref & \\
\hline Elderly & 1084 & $23.0 \%$ & $5.0 \%$ & & & $0.824(0.742 \sim 0.915)$ & 0.000 \\
\hline $\mathbf{T} 2$ & 1784 & & & 20.034 & 0.000 & & \\
\hline \multicolumn{8}{|l|}{ Age } \\
\hline Young & 809 & $39.6 \%$ & $9.4 \%$ & & & Ref & \\
\hline Elderly & 975 & $31.5 \%$ & $7.5 \%$ & & & $0.756(0.680 \sim 0.841)$ & 0.000 \\
\hline $\mathbf{T 3}$ & 1010 & & & 8.278 & 0.004 & & \\
\hline \multicolumn{8}{|l|}{ Age } \\
\hline Young & 492 & $38.8 \%$ & $10.1 \%$ & & & Ref & \\
\hline Elderly & 518 & $33.0 \%$ & $7.5 \%$ & & & $0.818(0.713 \sim 0.938)$ & 0.004 \\
\hline T4 & 2316 & & & 22.753 & 0.000 & & \\
\hline \multicolumn{8}{|l|}{ Age } \\
\hline Young & 1123 & $24.7 \%$ & $5.9 \%$ & & & Ref & \\
\hline Elderly & 1193 & $20.9 \%$ & $3.9 \%$ & & & $0.811(0.742 \sim 0.886)$ & 0.000 \\
\hline $\mathbf{T x}$ & 4375 & & & 51.779 & 0.000 & & \\
\hline \multicolumn{8}{|l|}{ Age } \\
\hline Young & 1850 & $24.5 \%$ & $4.3 \%$ & & & Ref & \\
\hline Elderly & 2525 & $18.5 \%$ & $3.4 \%$ & & & $0.784(0.733 \sim 0.838)$ & 0.000 \\
\hline
\end{tabular}

P values refer to comparison between two groups and were adjusted for years of diagnosis, sex, race, primary site, grade, histological type, surgery, marital status and clinical T stage.

proliferation and proteolytic activity in cancers [34]. Depression was strongly influenced by poor adherence to medical treatment.

This study has several limitations. First, the SEER database does not include information of therapeutic options such as detailed information of chemotherapy, targeted therapy, immunotherapy, recurrence and metastasis, which may also impact patients' prognosis [35]. Second, the SEER database is lack of detailed description of the organ metastasis (liver, lung, bone or brain). Third, since most patients did not receive operation, we used clinical $\mathrm{T}$ stage instead of pathological $\mathrm{T}$, which also may affect the analysis of prognosis in this study. Despite these limitations, we first reported that age was an independent prognostic factor in GC patients with M1. Further studies are needed to verify our findings.

\section{MATERIALS AND METHODS}

\section{Study population and data extracted}

The SEER database and SEER-stat software (SEER*Stat 8.3.2) were used to search GC patients with M1 between 2004 and 2013 with a known age $(\geq 18)$. Years of diagnosis, sex, race, primary site, grade, histological type, surgery, marital status, clinical T stage, and CSS were extracted from the SEER database. Histological types were limited to adenocarcinoma (8140/3), carcinoma (8010/3; $8020 / 3 ; 8021 / 3$ and $8145 / 3$ ) and signet ring cell carcinoma $(8490 / 3)$. Survival time was calculated from the date of diagnosis to the date of cancer-specific death. The exclusion criterions included: age $<18$, no evaluation of histological type, multiple malignant neoplasms, died within 30 days or information on CSS and survival months unavailable. 


\section{Statistical analysis}

Baseline characteristics were compared using the $\mathrm{X}^{2}$ test for nominal variables. Survival curves were generated using Kaplan-Meier analyses, and the differences between the curves were analyzed by log-rank test. Cox regression models were built for analysis of risk factors for survival outcomes. Statistical analyses were performed using the statistical software package SPSS for Windows, version 19.0 (SPSS Inc., Chicago, IL, USA). All P values were two-sided. $\mathrm{P}<0.05$ was considered statistically significant.

\section{Author contributions}

$\mathrm{ZH}$ and YS planned the study. HW and XiaoL calculated statistics and analyzed the data. CR and YW wrote the manuscript. XinL and WW supervised the entire project. All authors reviewed the manuscript.

\section{ACKNOWLEDGMENTS AND FUNDING}

This work was funded by National Youth Science Foundation (81402002). The authors acknowledged the efforts of the Surveillance, Epidemiology, and End Results (SEER) Program tumor registries in the creation of the SEER database. The interpretation and reporting of these data were the sole responsibility of the authors.

\section{CONFLICTS OF INTEREST}

The authors declare that they have no competing interest.

\section{REFERENCES}

1. Guimaraes RM, Muzi CD. Trend of mortality rates for gastric cancer in Brazil and regions in the period of 30 years (1980-2009). Arq Gastroenterol. 2012; 49: 184-8.

2. Ferlay J, Soerjomataram I, Dikshit R, Eser S, Mathers C, Rebelo M, Parkin DM, Forman D, Bray F. Cancer incidence and mortality worldwide: sources, methods and major patterns in GLOBOCAN 2012. Int J Cancer. 2015; 136: E359-86.

3. Siegel R, Ma J, Zou Z, Jemal A. Cancer statistics, 2014. CA Cancer J Clin. 2014; 64: 9-29.

4. Marques-Lespier JM, Gonzalez-Pons M, Cruz-Correa M. Current Perspectives on Gastric Cancer. Gastroenterol Clin North Am. 2016; 45: 413-28.

5. Choi YJ, Kim N. Gastric cancer and family history. Korean J Intern Med. 2016; 31: 1042-53.

6. Li Q, Cai G, Li D, Wang Y, Zhuo C, Cai S. Better long-term survival in young patients with non-metastatic colorectal cancer after surgery, an analysis of 69,835 patients in SEER database. PLoS One. 2014; 9: e93756.

7. Schellerer VS, Merkel S, Schumann SC, Schlabrakowski A, Fortsch T, Schildberg C, Hohenberger W, Croner RS.
Despite aggressive histopathology survival is not impaired in young patients with colorectal cancer : CRC in patients under 50 years of age. Int J Colorectal Dis. 2012; 27: 71-9.

8. Song P, Wu L, Jiang B, Liu Z, Cao K, Guan W. Age-specific effects on the prognosis after surgery for gastric cancer: A SEER population-based analysis. Oncotarget. 2016; 7: 48614-24. https://doi.org/10.18632/oncotarget.3129.

9. Chen J, Chen J, Xu Y, Long Z, Zhou Y, Zhu H, Wang Y, Shi Y. Impact of Age on the Prognosis of Operable Gastric Cancer Patients: An Analysis Based on SEER Database. Medicine (Baltimore). 2016; 95: e3944.

10. Anderson WF, Camargo MC, Fraumeni JF Jr, Correa P, Rosenberg PS, Rabkin CS. Age-specific trends in incidence of noncardia gastric cancer in US adults. JAMA. 2010; 303: 1723-8.

11. Park HJ, Ahn JY, Jung HY, Lim H, Lee JH, Choi KS, Kim DH, Choi KD, Song HJ, Lee GH, Kim JH. Clinical characteristics and outcomes for gastric cancer patients aged 18-30 years. Gastric Cancer. 2014; 17: 649-60.

12. Janssen CW Jr, Lie RT, Maartmann-Moe H, Matre R. The influence of age on the growth and spread of gastric carcinoma. Br J Cancer. 1991; 63: 623-5.

13. Yang D, Hendifar A, Lenz C, Togawa K, Lenz F, Lurje G, Pohl A, Winder T, Ning Y, Groshen S, Lenz HJ. Survival of metastatic gastric cancer: Significance of age, sex and race/ ethnicity. J Gastrointest Oncol. 2011; 2: 77-84.

14. Wong JE, Ito Y, Correa P, Peeters KC, van de Velde CJ, Sasako M, Macdonald J. Therapeutic strategies in gastric cancer. J Clin Oncol. 2003; 21: 267s-9s.

15. Zong L, Abe M, Seto Y, Ji J. The challenge of screening for early gastric cancer in China. Lancet. 2016; 388: 2606.

16. Lin Y, Ueda J, Kikuchi S, Totsuka Y, Wei WQ, Qiao YL, Inoue M. Comparative epidemiology of gastric cancer between Japan and China. World J Gastroenterol. 2011; 17 : 4421-8.

17. Ben-Ishay O, Brauner E, Peled Z, Othman A, Person B, Kluger Y. Diagnosis of colon cancer differs in younger versus older patients despite similar complaints. Isr Med Assoc J. 2013; 15: 284-7.

18. Park JC, Lee YC, Kim JH, Kim YJ, Lee SK, Hyung WJ, Noh $\mathrm{SH}$, Kim CB. Clinicopathological aspects and prognostic value with respect to age: an analysis of 3,362 consecutive gastric cancer patients. J Surg Oncol. 2009; 99: 395-401.

19. Nakamura R, Saikawa Y, Takahashi T, Takeuchi H, Asanuma H, Yamada Y, Kitagawa Y. Retrospective analysis of prognostic outcome of gastric cancer in young patients. Int J Clin Oncol. 2011; 16: 328-34.

20. Smith BR, Stabile BE. Extreme aggressiveness and lethality of gastric adenocarcinoma in the very young. Arch Surg. 2009; 144: 506-10.

21. Goodwin RA, Asmis TR. Overview of systemic therapy for colorectal cancer. Clin Colon Rectal Surg. 2009; 22: 251-6.

22. Chew $\mathrm{MH}, \mathrm{Koh} \mathrm{PK}, \mathrm{Ng} \mathrm{KH}, \mathrm{Eu} \mathrm{KW}$. Improved survival in an Asian cohort of young colorectal cancer patients: 
an analysis of 523 patients from a single institution. Int $\mathrm{J}$ Colorectal Dis. 2009; 24: 1075-83.

23. Kubota T, Hiki N, Sano T, Nomura S, Nunobe S, Kumagai K, Aikou S, Watanabe R, Kosuga T, Yamaguchi T. Prognostic significance of complications after curative surgery for gastric cancer. Ann Surg Oncol. 2014; 21: 891-8.

24. Sierzega M, Kolodziejczyk P, Kulig J, Polish Gastric Cancer Study G. Impact of anastomotic leakage on longterm survival after total gastrectomy for carcinoma of the stomach. Br J Surg. 2010; 97: 1035-42.

25. Adkins RB Jr, DeLozier JB, McKnight WG, Waterhouse G. Carcinoma of the colon in patients 35 years of age and younger. Am Surg. 1987; 53: 141-5.

26. Taylor MC, Pounder D, Ali-Ridha NH, Bodurtha A, MacMullin EC. Prognostic factors in colorectal carcinoma of young adults. Can J Surg. 1988; 31: 150-3.

27. Cusack JC, Giacco GG, Cleary K, Davidson BS, Izzo F, Skibber J, Yen J, Curley SA. Survival factors in 186 patients younger than 40 years old with colorectal adenocarcinoma. J Am Coll Surg. 1996; 183: 105-12.

28. Li Q, Gan L, Liang L, Li X, Cai S. The influence of marital status on stage at diagnosis and survival of patients with colorectal cancer. Oncotarget. 2015; 6: 7339-47. https://doi. org/10.18632/oncotarget.9548.
29. Jin JJ, Wang W, Dai FX, Long ZW, Cai H, Liu XW, Zhou Y, Huang H, Wang YN. Marital status and survival in patients with gastric cancer. Cancer Med. 2016; 5: 1821-9.

30. Mustafa M, Carson-Stevens A, Gillespie D, Edwards AG. Psychological interventions for women with metastatic breast cancer. Cochrane Database Syst Rev. 2013: CD004253.

31. Goodwin JS, Zhang DD, Ostir GV. Effect of depression on diagnosis, treatment, and survival of older women with breast cancer. J Am Geriatr Soc. 2004; 52: 106-11.

32. Goldzweig G, Andritsch E, Hubert A, Brenner B, Walach N, Perry S, Baider L. Psychological distress among male patients and male spouses: what do oncologists need to know? Ann Oncol. 2010; 21: 877-83.

33. Gallo LC, Troxel WM, Matthews KA, Kuller LH. Marital status and quality in middle-aged women: Associations with levels and trajectories of cardiovascular risk factors. Health Psychol. 2003; 22: 453-63.

34. Ferrara N, Davis-Smyth T. The biology of vascular endothelial growth factor. Endocr Rev. 1997; 18: 4-25.

35. Li Q, Zhuo C, Cai G, Zheng H, Li D, Cai S. Pathological features and survival outcomes of young patients with operable colon cancer: are they homogeneous? PLoS One. 2014; 9: e102004. 